Article

\title{
Activity of Saponins from Medicago Species against Phytoparasitic Nematodes
}

\author{
Trifone D'Addabbo ${ }^{1, *} \mathbb{0}$, Maria Pia Argentieri ${ }^{2}$, Jerzy Żuchowski ${ }^{3}{ }^{\oplus}$, Elisa Biazzi ${ }^{4}$, \\ Aldo Tava ${ }^{4}\left(\mathbb{D}\right.$, Wieslaw Oleszek ${ }^{3}(\mathbb{D})$ and Pinarosa Avato ${ }^{2} \mathbb{D}$ \\ 1 Institute for Sustainable Plant Protection, National Council of Research, 70125 Bari, Italy \\ 2 Department of Pharmacy-Drug Sciences, University of Bari Aldo Moro, 70125 Bari, Italy; \\ mariapia.argentieri@uniba.it (M.P.A.); pinarosa.avato@uniba.it (P.A.) \\ 3 Department of Biochemistry, Institute of Soil Science and Plant Cultivation-State Research Institute, \\ 24-100 Pulawi, Poland; jzuchowski@iung.pulawy.pl (J.Ż.); wo@iung.pulawy.pl (W.O.) \\ 4 CREA-Research Centre for Animal Production and Acquaculture, 26900 Lodi, Italy; \\ elisa.biazzi@crea.gov.it (E.B.); aldo.tava@crea.gov.it (A.T.) \\ * Correspondence: trifone.daddabbo@ipsp.cnr.it
}

Received: 16 March 2020; Accepted: 31 March 2020; Published: 2 April 2020

\begin{abstract}
Content of bioactive saponins of Medicago species suggests that they may also exert, as previously demonstrated on $M$. sativa, nematicidal properties exploitable for the formulation of new products for sustainable phytoparasitic nematode management. This study was addressed to highlight the bioactivity of saponins from five different Medicago species still poorly known for their biological efficacy, i.e., M. heyniana, M. hybrida, M. lupulina, M. murex and M. truncatula, against the plant parasitic nematodes Meloidogyne incognita, Xiphinema index and Globodera rostochiensis. The bioactivity of the extracts from the five Medicago species was assessed by in vitro assays on the juveniles (J2) and eggs of M. incognita and G. rostochiensis and the adult females of X. index. The suppressiveness to M. incognita of soil treatments with the Medicago plant biomasses was also investigated in a tomato experiment. The nematicidal activity of the five Medicago species was reported and discussed in relation to their phytochemical profile.
\end{abstract}

Keywords: Medicago; saponins; Meloidogyne incognita; Xiphinema index; Globodera rostochiensis; sustainable management

\section{Introduction}

Plants can be a large source of biocidal compounds potentially suitable to formulate new pesticides for sustainable management of plant pathogens and pests, also including phytoparasitic nematodes [1].

Presence of a variety of nematicidal phytochemicals in many botanical families has increasingly focused the attention of scientists and farmers to plant-derived nematicidal products [2,3]. Previous studies of our research group documented a strong activity on phytoparasitic nematodes for chemical constituents of extracts from Asteraceae and Brassicaceae plants, such as phenolics and glucosinolates [4,5], as well as for the essential oils from many aromatic and medicinal plants [6,7].

Saponins represent a wide group of specialized phytochemicals, consisting of a triterpene or steroid aglycone to which one or more sugar chains are attached, present in many plant families but particularly abundant in the Fabaceae plants [8]. Saponins were acknowledged for a wide range of biological activities, including a cytotoxic, antibiotic, anti-inflammatory and molluscicidal activity $[8,9]$. In addition, a high anthelmintic activity against gastrointestinal nematodes from donkey [10] and goats [11] was also proved for alfalfa (Medicago sativa L.) saponin mixtures.

The activity of saponins was also demonstrated on phytoparasitic nematodes, as affecting juvenile (J2) motility or egg and J2 viability of the root-knot nematodes Meloidogyne incognita Kofoid et White 
(Chitw) [12] and M. javanica Treub [13]. Moreover, saponin-rich extracts from Quillaja saponaria Molina were found to have significant nematicidal effects on other economically relevant phytonematode species, such as the dagger nematode Xiphinema index Thorne et Allen and the root lesion nematode Pratylenchus thornei Sher et Allen [14], as well as significantly reducing M. incognita infestation on field tomato (Solanum lycopersicum L.) or melon (Cucumis melo L.) crops [15].

The Medicago genus (Fabaceae family) contains 83 different herbaceous or shrub plant species, mainly distributed around the Mediterranean basin but also adapted to a large range of environmental conditions, which produce several specialized metabolites such as coumarins, flavonoids, naphtoquinones, alkaloids and also saponins [8,16-19].

Saponins from Medicago spp. are formed by complex mixtures of high molecular weight triterpene glycosides with medicagenic acid, hederagenin, zahnic acid, bayogenin and soyasapogenols A and B as the dominant aglycones [20,21]. Medicago saponins have been reported to possess a large spectrum of biological and pharmacological effects, such as cytotoxic, antitumor, fungicidal, molluscicidal, antibacterial and antiviral activities [8,22-24].

Previous studies of our research group assessed also the nematotoxic potential of active saponins obtained from various Medicago species. Argentieri et al. (2008) [25] described the nematicidal activity of saponins and derived prosapogenins and sapogenins from M. arborea, M. arabica and M. sativa against $X$. index, as well as in vitro bioassays demonstrated the activity of saponin mixtures from $M$. sativa against X. index, M. incognita and the potato cyst nematode Globodera rostochiensis Wollenweber [26]. The species M. heyniana Greuter, M. hybrida (Pourr.) Trautv., M. lupulina L., M. murex Willd. and M. truncatula Gaertn. are also widespread as fodder plants throughout the Mediterranean basin but, adversely to $M$. sativa, their biological activities were scarcely documented. The saponin content of these species suggests that they may also possess nematicidal properties exploitable for the formulation of new nematicidal products Therefore, a study was carried out to investigate the nematicidal activity of saponin-rich extracts from these five Medicago species against the phytoparasitic nematodes $M$. incognita, X. index and G. rostochiensis, as well as to assess the suppressiveness of soil amendments with their plant material to M. incognita on tomato.

\section{Results}

\subsection{Saponin Content and Composition}

Crude saponin content (\% of dry matter) of the five Medicago species under study is depicted in Table 1. Saponin amount differs in the five species, as ranging from $0.60 \pm 0.05 \%$ in M. truncatula to 1.62 $\pm 0.30 \%$ in M. heyniana. The crude saponin mixtures were purified at a highly pure grade $(80 \%-90 \%)$ by reverse-phase chromatography as here reported and then used for the chemical and biological studies.

Table 1. Saponin content (\% dry matter $\pm \mathrm{SD}$ ) in leaves of the five Medicago spp.

\begin{tabular}{cc}
\hline Medicago spp. & Saponin Content \\
\hline M. heyniana & $1.62 \pm 0.37$ \\
M. hybrida & $0.64 \pm 0.03$ \\
M. lupulina & $0.44 \pm 0.04$ \\
M. murex & $1.30 \pm 0.05$ \\
M. truncatula & $0.60 \pm 0.05$ \\
\hline
\end{tabular}

Preliminary investigation by TLC of the crude saponin extracts from the five Medicago species (Figure 1) revealed a complex chemical profile rich in several constituents, which overall indicated a quite different composition of the five crude extracts. Since the biological activity of saponins is related to both the aglycone moieties and the saccharidic parts, characterization of both aglycones and sugars has been performed. The evaluation of total aglycone moieties is reported in Table 2, while saponin composition (including preliminary evidence of their glycosidic moieties) is listed in Table 3. 


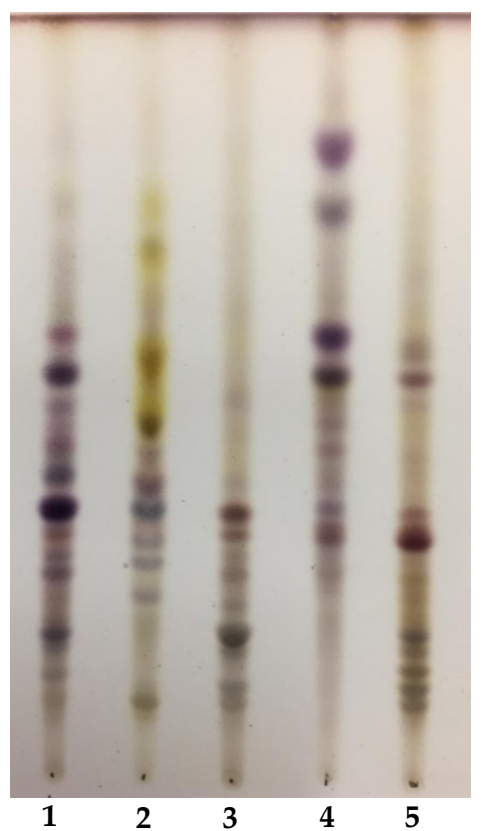

Figure 1. TLC of the five saponin extracts. 1: M. heyniana; 2: M. hybrida; 3: M. lupulina; 4: M. murex; 5: M. truncatula. Spots were visualized by Liebermann-Burckard reagent.

Table 2. Sapogenin composition (expressed as $\% \pm$ SD of total sapogenins) obtained by acid hydrolysis of saponins extracted by the five Medicago spp.

\begin{tabular}{cccccc}
\hline Sapogenin & M. heyniana & M. hybrida & M. lupulina & M. murex & M. truncatula \\
\hline Oleanolic acid & $4.2 \pm 1.3$ & $5.9 \pm 1.2$ & - & $2.8 \pm 0.3$ & - \\
Hederagenin & $37.6 \pm 2.3$ & $23.2 \pm 1.3$ & $<0.1$ & $43.7 \pm 0.9$ & - \\
Bayogenin & $39.4 \pm 0.8$ & $19.0 \pm 0.8$ & $2.6 \pm 0.1$ & $36.9 \pm 2.1$ & $0.8 \pm 0.1$ \\
Medicagenic acid & $2.8 \pm 0.7$ & $32.7 \pm 1.3$ & $46.4 \pm 1.7$ & $1.7 \pm 0.3$ & $18.1 \pm 0.8$ \\
Zanhic acid & $3.8 \pm 0.1$ & $13.6 \pm 0.7$ & $15.3 \pm 0.2$ & $0.7 \pm 0.2$ & $20.3 \pm 0.7$ \\
Soyasapogenol A & $0.9 \pm 0.3$ & - & $6.0 \pm 0.4$ & $1.0 \pm 0.2$ & $3.7 \pm 0.4$ \\
Soyasapogenol B & $6.4 \pm 1.7$ & $4.3 \pm 0.3$ & $24.8 \pm 0.6$ & $7.4 \pm 0.5$ & $51.6 \pm 1.3$ \\
\hline
\end{tabular}

Gas Chromatography/Flame-Ionization Detection (GC/FID) and GC/Mass Spectrometry (GC/MS) analyses of sapogenins released after acid hydrolysis of the crude obtained saponin mixtures allowed to identify the aglycone moieties composing the different saponins. As shown in Table 2, the five extracts have different compositions in terms of dominant aglycones. Hederagenin and bayogenin are the main sapogenis in M. heyniana $(37.6 \pm 2.3 \%$ and $39.4 \pm 0.8 \%$, respectively) and M. $\operatorname{murex}(47.3 \pm 0.9 \%$ and $36.9 \pm 2.1 \%$, respectively). The three aglycones hederagenin $(23.2 \pm 1.3 \%)$, bayogenin $(19.0 \pm 0.8 \%)$ and medicagenic acid (32.7 $\pm 1.3 \%)$ are dominant in $M$. hybrida, while M. lupulina is characterized by $46.4 \pm 1.7 \%$ of medicagenic acid, $15.3 \pm 0.2 \%$ of zanhic acid and $24.8 \% \pm 0.6 \%$ of soyasapogenol $\mathrm{B}$. This compound represents $51.6 \pm 1.3 \%$ of the total in $M$. truncatula, with medicagenic acid $(18.1 \pm 0.8 \%)$ and zanhic acid $(20.3 \pm 0.7 \%)$ as the other two main aglycones. 
Table 3. The most abundant tentatively identified saponins in the five Medicago spp. and their quantitative evaluation (\% of total saponins).

\begin{tabular}{|c|c|c|c|c|c|}
\hline & Rt & {$[\mathrm{M}-\mathrm{H}]^{-}$} & MS fragments & Tentative identification & $\%$ \\
\hline \multirow[t]{8}{*}{ M. heyniana } & 17.32 & 825 & $501[\mathrm{M}-\mathrm{H}-162-162]^{-}(\mathrm{Med})$ & Med-Hex-Hex & 2.6 \\
\hline & 17.80 & 795 & $663[\mathrm{M}-\mathrm{H}-162]^{-} ; 471[\mathrm{M}-\mathrm{H}-162-162]^{-}(\mathrm{Hed})$ & Hed-Hex-Hex & 1.6 \\
\hline & 19.14 & 765 & $603[\mathrm{M}-\mathrm{H}-162]^{-} ; 471[\mathrm{M}-\mathrm{H}-162-132]^{-}(\mathrm{Hed})$ & Hed-Pen-Hex & 20.3 \\
\hline & 19.26 & 765 & 633[M-H-132] $]^{-} ; 471[\mathrm{M}-\mathrm{H}-132-162]^{-}(\mathrm{Hed})$ & Hed-Pen-Hex & 6.8 \\
\hline & 20.08 & 811 & $767\left[\mathrm{M}-\mathrm{H}-\mathrm{CO}_{2}-\mathrm{H}_{2} \mathrm{O}\right]^{-} ; 487[\mathrm{M}-\mathrm{H}-162-162]^{-}$(Bayo) & Bayo-Hex-Hex & 16.4 \\
\hline & 21.39 & 811 & $649[\mathrm{M}-\mathrm{H}-162]^{-} ; 487[\mathrm{M}-\mathrm{H}-162-162]^{-}($Bayo $)$ & Bayo-Hex-Hex & 5.5 \\
\hline & 22.06 & 941 & $\begin{array}{c}879\left[\mathrm{M}-\mathrm{H}-\mathrm{CO}_{2}-\mathrm{H}_{2} \mathrm{O}\right]^{-} ; 795[\mathrm{M}-\mathrm{H}-146]^{-} ; 633[\mathrm{M}-\mathrm{H}-146-162]^{-} \\
457[\mathrm{M}-\mathrm{H}-146-132-176]^{-}(\text {SoyaB })\end{array}$ & Soya B-HexA-Hex-dHex (SSI) & 5.2 \\
\hline & 23.62 & 765 & $603[\mathrm{M}-\mathrm{H}-162]^{-} ; 471[\mathrm{M}-\mathrm{H}-132-162]^{-}(\mathrm{Hed})$ & Hed-Pen-Hex & 7.5 \\
\hline \multirow[t]{9}{*}{ M. hybrida } & 15.14 & 1397 & $\begin{array}{c}1265[\mathrm{M}-\mathrm{H}-132]^{-} ; 1011\left[\mathrm{M}-\mathrm{H}-\mathrm{CO}_{2}-\mathrm{H}_{2} \mathrm{O}-162-162\right]^{-} \\
455\left[\mathrm{M}-\mathrm{H}-\mathrm{CO}_{2}-\mathrm{H}_{2} \mathrm{O}-162-162-146-146-132-132\right]^{-}\left(\mathrm{Zanh}-\mathrm{CO}_{2}-\mathrm{H}_{2} \mathrm{O}\right)\end{array}$ & Zanh-Hex-Hex-dHex-dHex-Pen-Pen & 24.1 \\
\hline & 15.98 & 1235 & $\begin{array}{c}1103[\mathrm{M}-\mathrm{H}-132]^{-} ; 1011\left[\mathrm{M}-\mathrm{H}-\mathrm{CO}_{2}-\mathrm{H}_{2} \mathrm{O}-162\right]^{-} \\
\text {455[M-H-CO } \\
\left.\text { - } \mathrm{H}_{2} \mathrm{O}-162-146-146-132-132\right]^{-}\left(\mathrm{Zanh}-\mathrm{CO}_{2}-\mathrm{H}_{2} \mathrm{O}\right)\end{array}$ & Zanh-Hex-dHex-dHex-Pen-Pen & 3.6 \\
\hline & 16.90 & 825 & $\begin{array}{c}663[\mathrm{M}-\mathrm{H}-162]^{-} ; 601\left[\mathrm{M}-\mathrm{H}-162-\mathrm{CO}_{2}-\mathrm{H}_{2} \mathrm{O}\right]^{-} \\
487[\mathrm{M}-\mathrm{H}-162-176]^{-}(\text {Bayo })\end{array}$ & Bayo-HexA-Hex & 7.0 \\
\hline & 17.19 & 971 & $\begin{array}{c}\text { 809[M-H-162] }]^{-} ; 633[\mathrm{M}-\mathrm{H}-162-176]^{-} \\
471[\mathrm{M}-\mathrm{H}-162-176-162]^{-}(\mathrm{Hed})\end{array}$ & Hed-HexA-Hex-Hex & 2.0 \\
\hline & 17.51 & 825 & $663[\mathrm{M}-\mathrm{H}-162]^{-} ; 501[\mathrm{M}-\mathrm{H}-162-162]^{-}(\mathrm{Med})$ & Med-Hex-Hex & 26.5 \\
\hline & 17.83 & 795 & $663[\mathrm{M}-\mathrm{H}-162]^{-} ; 471[\mathrm{M}-\mathrm{H}-162-162]^{-}(\mathrm{Hed})$ & Hed-Hex-Hex & 3.6 \\
\hline & 21.39 & 811 & $649[\mathrm{M}-\mathrm{H}-162]^{-} ; 487[\mathrm{M}-\mathrm{H}-162-162]^{-}($Bayo $)$ & Bayo-Hex-Hex & 4.3 \\
\hline & 22.05 & 941 & $\begin{array}{c}879\left[\mathrm{M}-\mathrm{H}-\mathrm{CO}_{2}-\mathrm{H}_{2} \mathrm{O}\right]^{-} ; 795[\mathrm{M}-\mathrm{H}-146]^{-} ; 633[\mathrm{M}-\mathrm{H}-146-162]^{-} \\
457[\mathrm{M}-\mathrm{H}-146-132-176]^{-}(\text {SoyaB })\end{array}$ & Soya B-HexA-Hex-dHex (SSI) & 1.2 \\
\hline & 22.56 & 795 & 633[M-H-162] $]^{-} ; 471[\mathrm{M}-\mathrm{H}-162-162]^{-}(\mathrm{Hed})$ & Hed-Hex-Hex & 4.0 \\
\hline \multirow[t]{5}{*}{ M. lupulina } & 15.90 & 1103 & 927[M-H-176] $]^{-} ; 909\left[\mathrm{M}-\mathrm{H}-176-\mathrm{H}_{2} \mathrm{O}\right]^{-} ; 517[\mathrm{M}-\mathrm{H}-176-410]^{-}(\mathrm{Zanh})$ & Zanh-HexA-dHex-Pen-Pen & 6.1 \\
\hline & 17.63 & 1219 & $\begin{array}{c}\text { 1043[M-H-176] }]^{-} ; 911[\mathrm{M}-\mathrm{H}-176-132]^{-} \\
501[\mathrm{M}-\mathrm{H}-176-132-410]^{-}(\mathrm{Med})\end{array}$ & Med-HexA-dHex-Pen-Pen-Pen & 9.2 \\
\hline & 17.87 & 1087 & 911[M-H-176] $]^{-} ; 501[\mathrm{M}-\mathrm{H}-176-410]^{-}($Medic) & Med-HexA-dHex-Pen-Pen & 45.8 \\
\hline & 21.96 & 911 & 765[M-H-146] $]^{-} ; 457[\mathrm{M}-\mathrm{H}-146-132-176]^{-}$(SoyaB) & Soya B-HexA-Pen-dHex & 13.0 \\
\hline & 22.05 & 941 & $\begin{array}{c}879\left[\mathrm{M}-\mathrm{H}-\mathrm{CO}_{2}-\mathrm{H}_{2} \mathrm{O}\right]^{-} ; 795[\mathrm{M}-\mathrm{H}-146]^{-} ; 633[\mathrm{M}-\mathrm{H}-146-162]^{-} \\
457[\mathrm{M}-\mathrm{H}-146-132-176]^{-}(\text {SoyaB })\end{array}$ & Soya B-HexA-Hex-dHex (SSI) & 7.2 \\
\hline
\end{tabular}


Table 3. Cont

\begin{tabular}{|c|c|c|c|c|c|}
\hline & Rt & {$[\mathrm{M}-\mathrm{H}]^{-}$} & MS fragments & Tentative identification & $\%$ \\
\hline \multirow[t]{7}{*}{ M. murex } & 17.10 & 971 & $\begin{array}{l}\text { 809[M-H-162] }]^{-} ; 663[\mathrm{M}-\mathrm{H}-162-146]^{-} \\
\quad 487[\mathrm{M}-\mathrm{H}-162-146-176]^{-} \text {(Bayo) }\end{array}$ & Bayo-HexA-dHex-Hex & 3.2 \\
\hline & 18.92 & 955 & $\begin{array}{l}\text { 793[M-H-162] }]^{-} ; 647[\mathrm{M}-\mathrm{H}-162-146]^{-} \\
\text {471[M-H-162-146-176] }\end{array}$ & Hed-HexA-Pen-Hex & 7.7 \\
\hline & 20.09 & 811 & 767[M-H-CO $\left.{ }_{2}-\mathrm{H}_{2} \mathrm{O}\right]^{-} ; 487[\mathrm{M}-\mathrm{H}-162-162]^{-}$(Bayo) & Bayo-Hex-Hex & 44.1 \\
\hline & 22.03 & 941 & $\begin{array}{c}879\left[\mathrm{M}-\mathrm{H}-\mathrm{CO}_{2}-\mathrm{H}_{2} \mathrm{O}\right]^{-} ; 795[\mathrm{M}-\mathrm{H}-146]^{-} ; 633[\mathrm{M}-\mathrm{H}-146-162]^{-} \\
457[\mathrm{M}-\mathrm{H}-146-132-176]^{-}(\text {SoyaB })\end{array}$ & Soya B-HexA-Hex-dHex (SSI) & 28.7 \\
\hline & 22.56 & 795 & $633[\mathrm{M}-\mathrm{H}-162]^{-} ; 471[\mathrm{M}-\mathrm{H}-162-162]^{-}(\mathrm{Hed})$ & Hed-Hex-Hex & 1.5 \\
\hline & 22.85 & 765 & $619[\mathrm{M}-\mathrm{H}-146]^{-} ; 487[\mathrm{M}-\mathrm{H}-146-132]^{-}$(Вауо $)$ & Bayo-dHex-Pen & 5.3 \\
\hline & 23.64 & 765 & $603[\mathrm{M}-\mathrm{H}-162]^{-} ; 471[\mathrm{M}-\mathrm{H}-132-162]^{-}(\mathrm{Hed})$ & Hed-Hex-Pen & 4.0 \\
\hline \multirow[t]{6}{*}{ M. truncatula } & 15.70 & 1383 & $\begin{array}{c}\text { 997[M-H-162-162-CO2-H2O }]^{-} ; 841[\mathrm{M}-\mathrm{H}-132-410]^{-} \\
455\left[\mathrm{M}-\mathrm{H}-\mathrm{CO}_{2}-\mathrm{H}_{2} \mathrm{O}-132-410-162-162\right]^{-}\left(\mathrm{Zanh}-\mathrm{CO}_{2}-\mathrm{H}_{2} \mathrm{O}\right)\end{array}$ & Zanh-Hex-Hex-Pen-dHex-Pen-Pen & 3.1 \\
\hline & 15.82 & 1383 & $\begin{array}{c}\text { 997[M-H-162-162-CO2-H2O }]^{-} ; 841[\mathrm{M}-\mathrm{H}-132-410]^{-} \\
455\left[\mathrm{M}-\mathrm{H}-\mathrm{CO}_{2}-\mathrm{H}_{2} \mathrm{O}-132-410-162-162\right]^{-}\left(\mathrm{Zanh}-\mathrm{CO}_{2}-\mathrm{H}_{2} \mathrm{O}\right)\end{array}$ & Zanh-Hex-Hex-Pen-dHex-Pen-Pen & 11.9 \\
\hline & 17.56 & 1367 & $\begin{array}{c}\text { 1235[M-H-132] }]^{-} ; 981\left[\mathrm{M}-\mathrm{H}-162-162-\mathrm{CO}_{2}-\mathrm{H}_{2} \mathrm{O}^{-}\right. \\
849\left[\mathrm{M}-\mathrm{H}-162-162-\mathrm{CO}_{2}-\mathrm{H}_{2} \mathrm{O}-132\right]^{-} ; 439\left(\mathrm{Med}-\mathrm{CO}_{2}-\mathrm{H}_{2} \mathrm{O}\right)\end{array}$ & Med-Hex-Hex-Pen-dHex-Pen-Pen & 8.9 \\
\hline & 17.87 & 1087 & 911[M-H-176 $]^{-} ; 677[\mathrm{M}-\mathrm{H}-410]^{-} ; 501[\mathrm{M}-\mathrm{H}-176-410]^{-}(\mathrm{Med})$ & Med-HexA-Pen-dHex-Pen & 2.3 \\
\hline & 21.96 & 911 & 765[M-H-146] $]^{-} ; 457[\mathrm{M}-\mathrm{H}-146-132-176]^{-}($SoyaB $)$ & Soya B-HexA-Pen-dHex & 9.0 \\
\hline & 22.05 & 941 & $\begin{array}{c}879\left[\mathrm{M}-\mathrm{H}-\mathrm{CO}_{2}-\mathrm{H}_{2} \mathrm{O}\right]^{-} ; 795[\mathrm{M}-\mathrm{H}-146]^{-} ; 633[\mathrm{M}-\mathrm{H}-146-162]^{-} ; \\
457[\mathrm{M}-\mathrm{H}-146-132-176]^{-}(\text {SoyaB })\end{array}$ & Soya B-HexA-Hex-dHex (SSI) & 54.2 \\
\hline
\end{tabular}


Elucidation of the chemical structure of the saponins making up the five extracts was achieved based on their ESI-MS fragmentation and chromatographic behavior compared with authentic samples already fully identified by the authors in previous works and literature data [8,27-32]. Saponins were tentatively identified based on molecular ion $[\mathrm{M}-\mathrm{H}]^{-}$, on key fragment ions and other MS observations. In general, the loss of $132 \mathrm{~m} / \mathrm{z}$ was indicative of pentose (e.g., arabinose, xylose, apiose), the loss of $146 \mathrm{~m} / \mathrm{z}$ was indicative of deoxyhexose (e.g., rhamnose), the loss of $162 \mathrm{~m} / \mathrm{z}$ was indicative of hexose (e.g., glucose, galactose) and the loss of m/z 176 was indicative of hexuronic acid (e.g., glucuronic acid). The most abundant tentatively identified saponins in the five extracts are listed in Table 3.

High molecular weight compounds, in particular glycosides of medicagenic and zanhic acids, were detected in higher amounts in M. hybrida, M. lupulina and M. truncatula extracts. Shorter sugar chain saponins (2-3 sugars in the molecule) are instead most abundant in M. heyniana and M. murex extracts and represent a characteristic trait of these species. Soyasaponin I, a common saponin in the Fabaceae family, was detected in all the extracts in low amounts, but it represents more than $50 \%$ of the total saponins in M. truncatula extract.

\subsection{Nematode Mortality Assay}

The five saponin raw mixtures were poorly active on $M$. incognita $J 2$ at the 125 and $250 \mu \mathrm{g} \mathrm{mL} \mathrm{L}^{-1}$ concentrations, as nil or negligible mortality rates occurred for all the Medicago species (Table 4). At the $500 \mu \mathrm{g} \mathrm{mL}^{-1}$ concentration, $J 2$ mortality was above $90 \%$ after $16 \mathrm{~h}$ exposure to saponin extract of $M$. hybrida and M. truncatula or to $8 \mathrm{~h}$ contact with the saponin extract of M. murex s.

Adversely, the same concentration x exposure time combination occurred in $46.2 \%$ and $82.2 \% \mathrm{~J} 2$ mortalities for M. heyniana and M. Lupulina, respectively. Mortality of M. incognita J2 also ranged $90 \%$ after $8 \mathrm{~h}$ permanence in the $1000 \mu \mathrm{g} \mathrm{mL}{ }^{-1}$ solutions of M. heyniana and M. hybrida.

Table 4. Mortality (\%) of M. incognita J2 after 4 to $24 \mathrm{~h}$ exposures to $125-1000 \mu \mathrm{g} \mathrm{mL}^{-1}$ solutions of the saponin extract from five different Medicago species (means $\pm \mathrm{SE}$ ).

\begin{tabular}{|c|c|c|c|c|}
\hline \multirow{2}{*}{$\begin{array}{l}\text { Concentration } \\
\qquad\left(\mu \mathrm{g} \mathrm{mL}^{-1}\right)\end{array}$} & \multicolumn{4}{|c|}{ Exposure Time (hours) } \\
\hline & 4 & 8 & 16 & 24 \\
\hline & \multicolumn{4}{|c|}{ M. heyniana } \\
\hline 125 & $0.8 \pm 0.2$ & $0.9 \pm 0.2$ & $46.4 \pm 1.7$ & $1.7 \pm 0.3$ \\
\hline 250 & $1.4 \pm 0.4$ & $1.5 \pm 0.6$ & $2.3 \pm 0.4$ & $2.0 \pm 0.6$ \\
\hline 500 & $1.5 \pm 0.5$ & $3.3 \pm 0.9$ & $46.0 \pm 3.9$ & $90.1 \pm 0.4$ \\
\hline 1000 & $1.3 \pm 0.3$ & $89.8 \pm 3.0$ & $91.1 \pm 2.3$ & $97.9 \pm 0.3$ \\
\hline \multirow[t]{2}{*}{ LC50 } & $\geq$ & 798 & 521 & 353 \\
\hline & \multicolumn{4}{|c|}{ M. hybrida } \\
\hline 125 & $1.6 \pm 0.4$ & $2.8 \pm 0.3$ & $3.4 \pm 0.4$ & $3.4 \pm 0.2$ \\
\hline 250 & $2.4 \pm 1.0$ & $3.4 \pm 0.2$ & $3.6 \pm 0.2$ & $3.7 \pm 0.2$ \\
\hline 500 & $2.4 \pm 0.6$ & $23.6 \pm 2.1$ & $95.9 \pm 1.0$ & $95.1 \pm 0.2$ \\
\hline 1000 & $3.4 \pm 0.9$ & $88.1 \pm 3.0$ & $96.8 \pm 0.5$ & $95.8 \pm 0.4$ \\
\hline \multirow[t]{2}{*}{ LC50 } & $\geq$ & 612 & 353 & 363 \\
\hline & \multicolumn{4}{|c|}{ M. lupulina } \\
\hline 125 & $0.5 \pm 0.2$ & $2.7 \pm 0.4$ & $3.3 \pm 0.4$ & $3.5 \pm 0.2$ \\
\hline 250 & $0.5 \pm 0.3$ & $3.7 \pm 0.5$ & $5.8 \pm 0.6$ & $6.1 \pm 0.4$ \\
\hline 500 & $4.3 \pm 0.5$ & $5.2 \pm 0.4$ & $82.2 \pm 1.7$ & $89.2 \pm 0.3$ \\
\hline 1000 & $7.8 \pm 1.0$ & $11.5 \pm 2.0$ & $89.3 \pm 3.1$ & $90.2 \pm 1.8$ \\
\hline \multirow[t]{2}{*}{ LC50 } & $\geq$ & $\geq$ & 422 & 404 \\
\hline & \multicolumn{4}{|c|}{ M. murex } \\
\hline 125 & $1.4 \pm 0.3$ & $1.9 \pm 0.7$ & $1.9 \pm 0.3$ & $2.1 \pm 0.5$ \\
\hline 250 & $1.5 \pm 0.4$ & $2.0 \pm 0.6$ & $2.2 \pm 0.7$ & $2.8 \pm 0.4$ \\
\hline 500 & $2.0 \pm 0.6$ & $91.3 \pm 3.6$ & $91.4 \pm 3.0$ & $92.1 \pm 0.2$ \\
\hline 1000 & $2.7 \pm 1.0$ & $92.3 \pm 1.4$ & $96.0 \pm 0.5$ & $95.5 \pm 0.6$ \\
\hline LC50 & $\geq$ & 414 & 383 & 385 \\
\hline
\end{tabular}


Table 4. Cont.

\begin{tabular}{|c|c|c|c|c|}
\hline \multirow{2}{*}{$\begin{array}{l}\text { Concentration } \\
\left(\mu \mathrm{g} \mathrm{mL}^{-1}\right)\end{array}$} & \multicolumn{4}{|c|}{ Exposure Time (hours) } \\
\hline & 4 & 8 & 16 & 24 \\
\hline & \multicolumn{4}{|c|}{ M. truncatula } \\
\hline 125 & $0.9 \pm 0.2$ & $1.6 \pm 0.4$ & $2.0 \pm 1.5$ & $4.4 \pm 0.4$ \\
\hline 250 & $1.1 \pm 0.1$ & $4.7 \pm 0.3$ & $4.8 \pm 0.1$ & $4.7 \pm 0.2$ \\
\hline 500 & $1.2 \pm 0.2$ & $33.3 \pm 3.8$ & $90.4 \pm 1.8$ & $90.6 \pm 0.3$ \\
\hline 1000 & $2.7 \pm 1.1$ & $38.3 \pm 1.3$ & $91.4 \pm 4.4$ & $95.5 \pm 1.8$ \\
\hline LC50 & $\geq$ & 1098 & 416 & 360 \\
\hline Oxamyl (1 mL L $\left.{ }^{-1}\right)$ & $2.8 \pm 0.3$ & $40.0 \pm 1.0$ & $88.2 \pm 0.5$ & $94.5 \pm 0.7$ \\
\hline Water & 0.0 & 0.0 & 0.0 & 0.0 \\
\hline LSD 0.05 & 1.7 & 4.7 & 5.0 & 1.9 \\
\hline
\end{tabular}

The 125 and $250 \mu \mathrm{g} \mathrm{mL}^{-1}$ solutions of saponin extract from M. heyniana, M. hybrida and $M$. truncatula poorly affected $X$. index females at all the exposure times, whereas a $26.8 \%$ mortality occurred after $8 \mathrm{~h}$ immersion in the $250 \mu \mathrm{g} \mathrm{mL}^{-1}$ solutions of M. lupulina and M. murex saponins (Table 5). The activity of M. hybrida and M. truncatula continued to be limited also at a $500 \mu \mathrm{g} \mathrm{mL}^{-1}$ concentration, as the mortaliy peaked only $23.3 \%$ at the longest exposure time. The same concentration resulted in $36.8 \%$ and $43.3 \%$ mortality after $16 \mathrm{~h}$ exposure to $M$. heyniana and $M$. murex saponins, respectively, or even in $93.3 \%$ mortality for the $8 \mathrm{~h}$ treatment with the M. lupulina saponin extract.

Table 5. Mortality (\%) of X. index adult females after 4 to 24 h exposures to $125-1000 \mu \mathrm{g} \mathrm{mL}^{-1}$ solutions of the saponin extract from five different Medicago species (means $\pm \mathrm{SE}$ ).

\begin{tabular}{|c|c|c|c|c|}
\hline \multirow{2}{*}{$\begin{array}{l}\text { Concentration } \\
\qquad\left(\mu g \mathrm{~mL}^{-1}\right)\end{array}$} & \multicolumn{4}{|c|}{ Exposure Time (hours) } \\
\hline & 4 & 8 & 16 & 24 \\
\hline & \multicolumn{4}{|c|}{ M. heyniana } \\
\hline 125 & 0.0 & 0.0 & 0.0 & $3.3 \pm 2.4$ \\
\hline 250 & 0.0 & 0.0 & 0.0 & $3.3 \pm 0.6$ \\
\hline 500 & 0.0 & $6.8 \pm 4.7$ & $36.8 \pm 4.1$ & $56.7 \pm 6.2$ \\
\hline 1000 & $40.0 \pm 4.1$ & $\begin{array}{c}66.8 \pm \\
10.3\end{array}$ & $80.0 \pm 6.2$ & 100 \\
\hline \multirow[t]{2}{*}{ LC50 } & $\geq$ & 856 & 611 & 544 \\
\hline & \multicolumn{4}{|c|}{ M. hybrida } \\
\hline 125 & 0.0 & 0.0 & 0.0 & $3.3 \pm 2.4$ \\
\hline 250 & 0.0 & $3.3 \pm 2.4$ & $3.3 \pm 2.4$ & $6.7 \pm 2.4$ \\
\hline 500 & 0.0 & $6.8 \pm 2.4$ & $13.3 \pm 2.4$ & $23.3 \pm 2.2$ \\
\hline 1000 & $3.2 \pm 2.4$ & $6.8 \pm 4.7$ & $30.0 \pm 4.1$ & $46.7 \pm 2.8$ \\
\hline \multirow[t]{2}{*}{ LC50 } & $\geq$ & $\geq$ & 1692 & 1165 \\
\hline & \multicolumn{4}{|c|}{ M. lupulina } \\
\hline 125 & $6.8 \pm 2.4$ & $6.8 \pm 2.4$ & $12.3 \pm 1.6$ & $6.7 \pm 2.4$ \\
\hline 250 & $20.0 \pm 8.2$ & $26.8 \pm 2.4$ & $33.3+2.4$ & $43.3 \pm 4.7$ \\
\hline 500 & $\begin{array}{c}56.7 \pm \\
16.5\end{array}$ & $93.3 \pm 2.4$ & $96.7 \pm 2.5$ & 100 \\
\hline 1000 & $100 \pm 0$ & 100 & 100 & 100 \\
\hline \multirow[t]{2}{*}{ LC50 } & 457 & 274 & 241 & 273 \\
\hline & \multicolumn{4}{|c|}{ M. murex } \\
\hline 125 & 0.0 & $13.3 \pm 2.4$ & $26.7 \pm 2.4$ & $36.7 \pm 4.7$ \\
\hline 250 & 0.0 & $26.8 \pm 6.2$ & $40.0 \pm 4.7$ & $43.3 \pm 6.2$ \\
\hline 500 & 0.0 & $33.3 \pm 2.4$ & $43.3 \pm 2.4$ & $50.0 \pm 4.1$ \\
\hline 1000 & $16.8 \pm 4.7$ & $43.3 \pm 2.4$ & $63.3 \pm 4.1$ & $83.3 \pm 2.4$ \\
\hline LC50 & $\geq$ & 1357 & 536 & 295 \\
\hline
\end{tabular}


Table 5. Cont.

\begin{tabular}{|c|c|c|c|c|}
\hline \multirow{2}{*}{$\begin{array}{l}\text { Concentration } \\
\qquad\left(\mu \mathrm{g} \mathrm{mL}^{-1}\right)\end{array}$} & \multicolumn{4}{|c|}{ Exposure Time (hours) } \\
\hline & 4 & 8 & 16 & 24 \\
\hline & \multicolumn{4}{|c|}{ M. truncatula } \\
\hline 125 & 0.0 & 0.0 & 0.0 & 0.0 \\
\hline 250 & $6.8 \pm 2.4$ & $6.8 \pm 2.4$ & $6.7 \pm 2.3$ & $10.0 \pm 1.1$ \\
\hline 500 & $13.3 \pm 4.7$ & $16.8 \pm 4.7$ & $23.3 \pm 3.3$ & $23.3 \pm 2.4$ \\
\hline 1000 & $30.0 \pm 7.1$ & $86.8 \pm 4.7$ & $96.7 \pm 2.4$ & $97.6 \pm 3.2$ \\
\hline LC50 & 2228 & 628 & 525 & 498 \\
\hline Oxamyl $\left(1 \mathrm{~mL} \mathrm{~L}^{-1}\right)$ & $38.5 \pm 4.1$ & $80.0 \pm 4.1$ & $95.0 \pm 2.9$ & 100 \\
\hline Water & 0.0 & 0.0 & 0.0 & 0.0 \\
\hline LSD 0.05 & 14.5 & 14.3 & 15.6 & 18.4 \\
\hline
\end{tabular}

An almost complete nematode mortality was recorded after $24 \mathrm{~h}$ treatment with the highest concentration of $M$. heyniana, M. lupulina and $M$. truncatula saponins, whereas the same concentration $\mathrm{x}$ time combination peaked 83.3 and $46.7 \%$ mortality rates for the saponins of M. murex and M. hybrida, respectively. Interestingly, the $1000 \mu \mathrm{g} \mathrm{mL}^{-1}$ concentration of $\mathrm{M}$. lupulina caused total nematode mortality even after $4 \mathrm{~h}$ treatment.

The $J 2$ of G. rostochiensis was more sensitive to the five Medicago extracts than the other two nematode species. After a $4 \mathrm{~h}$ exposure, mortality rates ranged from $23 \%$ to $27 \%$ at the lowest concentration of all saponin mixtures, except for that of $M$. truncatula, and varied from 39 to $47 \%$ for the $1000 \mu \mathrm{g} \mathrm{mL}^{-1}$ solutions (Table 6). The higher sensitivity of G. rostochiensis J2 was confirmed also at the longer exposure times, as after $24 \mathrm{~h}$ immmersion in $125 \mu \mathrm{g} \mathrm{mL} \mathrm{L}^{-1}$ solutions, the mortality rates ranged from about $56 \%$ of $M$. truncatula to $60-64 \%$ of M. murex and M. heyniana, respectively, up to more than $75 \%$ for M. hybrida and M. lupulina. As also found for M. incognita and X. index, the highest concentration of the five saponin mixtures generally resulted in $J 2$ mortality rates not significantly different from those of Oxamyl.

Table 6. Mortality (\%) of G. rostochiensis J2 after 4 to $24 \mathrm{~h}$ exposures to $125-1000 \mu \mathrm{g} \mathrm{mL} \mathrm{m}^{-1}$ solutions of the saponin extract from five different Medicago species (means \pm SE).

\begin{tabular}{ccccc}
\hline Concentration & \multicolumn{5}{c}{ Exposure Time (hours) } \\
\cline { 2 - 5 }$\left(\mu \mathbf{g ~ m L}^{-1}\right)$ & $\mathbf{4}$ & $\mathbf{8}$ & $\mathbf{1 6}$ & $\mathbf{2 4}$ \\
\hline \multicolumn{5}{c}{ M. heyniana } \\
125 & $22.7 \pm 1.7$ & $39.5 \pm 1.3$ & $42.7 \pm 0.6$ & $64.5 \pm 1.4$ \\
250 & $29.6 \pm 3.2$ & $43.0 \pm 2.3$ & $47.9 \pm 1.0$ & $72.1 \pm 3.0$ \\
500 & $37.7 \pm 6.0$ & $48.5 \pm 4.0$ & $67.8 \pm 4.0$ & $80.1 \pm 1.7$ \\
1000 & $38.9 \pm 5.6$ & $55.8 \pm 4.6$ & $80.8 \pm 3.9$ & $88.2 \pm 1.4$ \\
LC50 & 2648 & 546 & 212 & 52 \\
& \multicolumn{4}{c}{ M. hybrida } \\
125 & $25.8 \pm 1.5$ & $29.0 \pm 1.3$ & $62.9 \pm 4.4$ & $76.3 \pm 4.5$ \\
250 & $28.7 \pm 2.9$ & $32.2 \pm 2.6$ & $64.0 \pm 4.0$ & $77.0 \pm 1.2$ \\
500 & $33.6 \pm 1.3$ & $37.5 \pm 1.0$ & $65.0 \pm 5.6$ & $87.0 \pm 4.0$ \\
1000 & $44.7 \pm 4.0$ & $47.9 \pm 3.8$ & $82.6 \pm 4.6$ & $87.8 \pm 1.5$ \\
LC50 & 2191 & 1538 & 54 & 9 \\
& \multicolumn{4}{c}{ M. lupulina } \\
125 & $23.1 \pm 0.6$ & $27.4 \pm 2.2$ & $73.0 \pm 4.8$ & $75.5 \pm 4.7$ \\
250 & $29.3 \pm 1.9$ & $30.0 \pm 1.5$ & $79.3 \pm 4.4$ & $79.8 \pm 1.8$ \\
500 & $33.4 \pm 3.7$ & $41.9 \pm 2.7$ & $85.6 \pm 4.8$ & $85.4 \pm 3.2$ \\
1000 & $40.7 \pm 2.7$ & $47.8 \pm 1.4$ & $86.9 \pm 1.8$ & $86.7 \pm 1.9$ \\
LC50 & 2868 & 1215 & 10 & 5 \\
\hline
\end{tabular}


Table 6. Cont.

\begin{tabular}{|c|c|c|c|c|}
\hline \multirow{2}{*}{$\begin{array}{l}\text { Concentration } \\
\left(\mu \mathrm{g} \mathrm{mL}^{-1}\right)\end{array}$} & \multicolumn{4}{|c|}{ Exposure Time (hours) } \\
\hline & 4 & 8 & 16 & 24 \\
\hline & \multicolumn{4}{|c|}{ M. murex } \\
\hline 125 & $26.6 \pm 0.9$ & $29.3 \pm 2.8$ & $34.5 \pm 2.4$ & $60.1 \pm 4.8$ \\
\hline 250 & $31.1 \pm 2.1$ & $34.1 \pm 1.6$ & $47.1 \pm 1.1$ & $61.8 \pm 2.7$ \\
\hline 500 & $35.4 \pm 2.0$ & $44.9 \pm 0.7$ & $57.1 \pm 0.9$ & $72.6 \pm 2.4$ \\
\hline 1000 & $46.8 \pm 1.8$ & $54.5 \pm 4.2$ & $72.7 \pm 1.1$ & $84.9 \pm 2.6$ \\
\hline \multirow[t]{2}{*}{ LC50 } & 1688 & 751 & 302 & 84 \\
\hline & \multicolumn{4}{|c|}{ M. truncatula } \\
\hline 125 & $10.8 \pm 3.4$ & $24.9 \pm 1.3$ & $33.4 \pm 1.0$ & $55.8 \pm 5.0$ \\
\hline 250 & $22.7 \pm 2.1$ & $30.0 \pm 2.1$ & $52.6 \pm 1.6$ & $62.4 \pm 2.0$ \\
\hline 500 & $25.7 \pm 3.3$ & $43.0 \pm 4.2$ & $67.8 \pm 0.9$ & $75.1 \pm 1.2$ \\
\hline 1000 & $40.8 \pm 3.4$ & $51.7 \pm 4.1$ & $76.6 \pm 2.1$ & $85.6 \pm 2.3$ \\
\hline LC50 & 1755 & 895 & 245 & 104 \\
\hline Oxamyl $\left(1 \mathrm{~mL} \mathrm{~L}^{-1}\right)$ & $34.5 \pm 1.7$ & $61.7 \pm 1.3$ & $78.3 \pm 0.5$ & $88.0 \pm 0.7$ \\
\hline Water & $0.0 \pm$ & 0.0 & 0.0 & 0.0 \\
\hline LSD 0.05 & 8.3 & 6.6 & 12.9 & 8.0 \\
\hline
\end{tabular}

\subsection{Egg Hatchability Bioassay}

A one- or two-week permanence of M. incognita egg masses in all the saponin solutions of the five Medicago species significantly reduced the percentage egg hatch compared to the water control and, at the $1000 \mu \mathrm{g} \mathrm{mL}^{-1}$ concentration, also to the Oxamyl solution (Table 7). Egg hatch ranged from $52-47 \%$ to $39-18 \%$ at $125 \mu \mathrm{g} \mathrm{mL} \mathrm{m}^{-1}$ and $1000 \mu \mathrm{g} \mathrm{mL}-1$, respectively, at the one-week exposure, and was furtherly reduced after a two-week treatment, ranging from $39-32 \%$ at $125 \mu \mathrm{g} \mathrm{mL}^{-1}$ ) to $21-10 \%$ $1000 \mu \mathrm{g} \mathrm{mL}{ }^{-1}$. The hatchability of G. rostochiensis eggs was never reduced by one-week treatment of the cysts with any of the saponin solutions, as results were not significantly different from the $0.6 \mathrm{mM}$ sodium metavanadate control for the $125-500 \mu \mathrm{g} \mathrm{mL} \mathrm{m}^{-1}$ solutions of $M$. heyniana saponin extract and the lowest concentration of M. lupulina, M. murex and M. truncatula extracts.

Table 7. Hatchability (\%) of M. incognita and G. rostochiensis eggs after a 1- or 2-week exposure of egg masses and cysts, respectively, to $125-1000 \mu \mathrm{g} \mathrm{mL} \mathrm{m}^{-1}$ solutions of the saponin extract from five different Medicago species (means $\pm \mathrm{SE}$ ).

\begin{tabular}{|c|c|c|c|c|}
\hline \multirow{2}{*}{$\begin{array}{l}\text { Concentration } \\
\qquad\left(\mu \mathrm{g} \mathrm{mL}^{-1}\right)\end{array}$} & \multicolumn{2}{|c|}{1 week } & \multicolumn{2}{|c|}{2 weeks } \\
\hline & M. incognita & G. rostochiensis & M. incognita & G. rostochiensis \\
\hline & \multicolumn{4}{|c|}{ M. heyniana } \\
\hline 125 & $47.0 \pm 2.1$ & $33.0 \pm 0.6$ & $38.7 \pm 2.2$ & $35.7 \pm 1.2$ \\
\hline 250 & $33.0 \pm 1.0$ & $39.3 \pm 1.2$ & $26.0 \pm 1.5$ & $43.3 \pm 0.9$ \\
\hline 500 & $24.3 \pm 0.9$ & $42.0 \pm 2.1$ & $17.7 \pm 1.2$ & $48.0 \pm 2.1$ \\
\hline \multirow[t]{2}{*}{1000} & $24.0 \pm 1.1$ & $46.7 \pm 3.2$ & $15.0 \pm 0.6$ & $69.3 \pm 2.2$ \\
\hline & \multicolumn{4}{|c|}{ M. hybrida } \\
\hline 125 & $49.0 \pm 1.5$ & $44.0 \pm 1.5$ & $32.0 \pm 1.2$ & $51.0 \pm 2.5$ \\
\hline 250 & $39.7 \pm 2.3$ & $59.0 \pm 1.0$ & $23.3 \pm 0.9$ & $61.7 \pm 1.8$ \\
\hline 500 & $28.7 \pm 1.4$ & $71.7 \pm 0.7$ & $18.7 \pm 0.7$ & $85.0 \pm 1.7$ \\
\hline \multirow[t]{2}{*}{1000} & $18.3 \pm 0.9$ & $80.0 \pm 9.3$ & $16.0 \pm 0.6$ & $95.7 \pm 1.3$ \\
\hline & \multicolumn{4}{|c|}{ M. lupulina } \\
\hline 125 & $51.7 \pm 1.4$ & $36.3 \pm 0.3$ & $42.3 \pm 0.7$ & $37.3 \pm 0.3$ \\
\hline 250 & $46.7 \pm 0.3$ & $47.3 \pm 0.7$ & $38.0 \pm 1.0$ & $49.0 \pm 0.0$ \\
\hline 500 & $34.7 \pm 0.3$ & $55.7 \pm 0.9$ & $26.7 \pm 0.7$ & $60.7 \pm 3.5$ \\
\hline 1000 & $20.3 \pm 0.9$ & $80.7 \pm 0.9$ & $10.0 \pm 0.6$ & $93.3 \pm 2.0$ \\
\hline
\end{tabular}


Table 7. Cont.

\begin{tabular}{|c|c|c|c|c|}
\hline \multirow{2}{*}{$\begin{array}{l}\text { Concentration } \\
\left(\mu \mathrm{g} \mathrm{mL}^{-1}\right)\end{array}$} & \multicolumn{2}{|c|}{1 week } & \multicolumn{2}{|c|}{2 weeks } \\
\hline & M. incognita & G. rostochiensis & M. incognita & G. rostochiensis \\
\hline & \multicolumn{4}{|c|}{ M. murex } \\
\hline 125 & $51.0 \pm 1.0$ & $38.3 \pm 0.9$ & $35.3 \pm 1.8$ & $45.3 \pm 2.0$ \\
\hline 250 & $41.7 \pm 1.2$ & $53.0 \pm 2.3$ & $28.7 \pm 0.9$ & $61.3 \pm 0.9$ \\
\hline 500 & $33.7 \pm 0.9$ & $60.0 \pm 2.0$ & $23.3 \pm 0.7$ & $72.3 \pm 0.3$ \\
\hline \multirow[t]{2}{*}{1000} & $21.7 \pm 0.7$ & $67.7 \pm 1.9$ & $19.0 \pm 1.0$ & $93.3 \pm 3.3$ \\
\hline & \multicolumn{4}{|c|}{ M. truncatula } \\
\hline 125 & $52.0 \pm 2.1$ & $39.3 \pm 1.8$ & $39.0 \pm 0.6$ & $45.3 \pm 1.4$ \\
\hline 250 & $45.3 \pm 0.3$ & $46.7 \pm 2.2$ & $34.0 \pm 1.2$ & $55.7 \pm 1.8$ \\
\hline 500 & $39.7 \pm 1.5$ & $52.0 \pm 4.5$ & $23.0 \pm 1.5$ & $62.7 \pm 0.9$ \\
\hline 1000 & $39.0 \pm 1.2$ & $71.0 \pm 2.1$ & $21.3 \pm 0.7$ & $96.7 \pm 1.2$ \\
\hline Oxamyl (1 mL L $\left.{ }^{-1}\right)$ & $29.3 \pm 1.2$ & $25.3 \pm 0.9$ & $20.0 \pm 1.2$ & $15.7 \pm 0.9$ \\
\hline Control & $56.3 \pm 0.7$ & $35.7 \pm 1.8$ & $56.3 \pm 0.7$ & $35.7 \pm 1.8$ \\
\hline LSD 0.05 & 3.6 & 7.6 & 3.1 & 5.1 \\
\hline
\end{tabular}

Adversely, all the other one-week treatments with the saponin in solutions caused a significant increase of G. rostochiensis egg hatch, with an $80 \%$ peak for the $1000 \mu \mathrm{g} \mathrm{mL}^{-1}$ solutions of M. hybrida and M. lupulina saponins. This significant egg hatch increase was extended, according to a dose-related relationship, also to almost all the two-week treatments with the saponin solutions, with $93-96 \%$ percentage hatch peaks at the $1000 \mu \mathrm{g} \mathrm{mL}^{-1}$ concentrations of saponins from all the Medicago species except for M. heyniana.

\subsection{Experiment in Soil}

Compared to the non-treated control, all the soil amendments with biomasses from the five Medicago species significantly suppressed $M$. incognita multiplication and gall formation on tomato roots as well as the final nematode population in soil (Table 8). Moreover, almost all the highest amendment rates were not significantly different, or even lower, than Oxamyl.

Table 8. Effect of soil amendments with dry plant biomass of the five tested Medicago species on the infestation of the root-knot nematode M. incognita and on the growth of tomato cv. Regina (means \pm $\mathrm{SE})$.

\begin{tabular}{|c|c|c|c|c|c|}
\hline \multirow{2}{*}{$\begin{array}{l}\text { Amendment Rate } \\
\qquad\left(\mathrm{g} \mathrm{kg}^{-1} \text { soil) }\right.\end{array}$} & \multicolumn{2}{|c|}{ Nematode Eggs and J2 } & \multirow{2}{*}{$\begin{array}{l}\text { Root Gall } \\
\text { Index (0-5) }\end{array}$} & \multicolumn{2}{|c|}{ Plant Fresh Weight (g) } \\
\hline & $\mathrm{g}^{-1}$ roots $(x \mathrm{1000})$ & $\left(\mathrm{mL}^{-1}\right.$ soil) & & Aerial Parts & Roots \\
\hline & & \multicolumn{4}{|c|}{ M. heyniana } \\
\hline 10 & $5.2 \pm 0.2$ & $14.0 \pm 0.5$ & $4.4 \pm 0.2$ & $32.4 \pm 1.3$ & $8.8 \pm 0.2$ \\
\hline 20 & $3.5 \pm 0.1$ & $6.8 \pm 0.4$ & $4.0 \pm 0.3$ & $43.0 \pm 0.7$ & $12.8 \pm 0.5$ \\
\hline \multirow[t]{2}{*}{40} & $1.5 \pm 0.1$ & $4.4 \pm 0.4$ & $2.2 \pm 0.4$ & $51.4 \pm 1.0$ & $20.4 \pm 0.6$ \\
\hline & & \multicolumn{4}{|c|}{ M. hybrida } \\
\hline 10 & $5.1 \pm 0.4$ & $13.6 \pm 0.2$ & $4.4 \pm 0.4$ & $30.2 \pm 1.6$ & $5.6 \pm 0.4$ \\
\hline 20 & $3.6 \pm 0.2$ & $6.2 \pm 0.5$ & $3.8 \pm 0.2$ & $38.4 \pm 0.8$ & $10.8 \pm 0.7$ \\
\hline \multirow[t]{2}{*}{40} & $1.6 \pm 0.3$ & $4.0 \pm 0.3$ & $3.2 \pm 0.2$ & $47.4 \pm 2.1$ & $15.8 \pm 0.4$ \\
\hline & & \multicolumn{4}{|c|}{ M. lupulina } \\
\hline 10 & $7.0 \pm 0.3$ & $7.2 \pm 0.4$ & $4.6 \pm 0.2$ & $33.2 \pm 1.1$ & $9.0 \pm 0.3$ \\
\hline 20 & $2.8 \pm 0.4$ & $6.4 \pm 0.5$ & $3.6 \pm 0.2$ & $42.6 \pm 1.4$ & $11.6 \pm 0.2$ \\
\hline \multirow[t]{2}{*}{40} & $0.9 \pm 0.01$ & $5.0 \pm 0.3$ & $2.4 \pm 0.3$ & $56.6 \pm 1.2$ & $19.2 \pm 1.4$ \\
\hline & & \multicolumn{4}{|c|}{ M. murex } \\
\hline 10 & $5.4 \pm 0.3$ & $10.0 \pm 0.5$ & $3.8 \pm 0.4$ & $35.4 \pm 0.8$ & $5.0 \pm 0.3$ \\
\hline 20 & $3.1 \pm 0.2$ & $4.0 \pm 0.5$ & $2.8 \pm 0.5$ & $43.6 \pm 1.2$ & $7.4 \pm 0.2$ \\
\hline 40 & $1.5 \pm 0.1$ & $1.8 \pm 0.2$ & $2.2 \pm 0.4$ & $60.8 \pm 1.5$ & $14.4 \pm 0.8$ \\
\hline
\end{tabular}


Table 8. Cont.

\begin{tabular}{|c|c|c|c|c|c|}
\hline \multirow{2}{*}{$\begin{array}{l}\text { Amendment Rate } \\
\qquad\left(\mathrm{g} \mathrm{kg}^{-1} \text { soil) }\right.\end{array}$} & \multicolumn{2}{|c|}{ Nematode Eggs and J2 } & \multirow{2}{*}{$\begin{array}{l}\text { Root Gall } \\
\text { Index (0-5) }\end{array}$} & \multicolumn{2}{|c|}{ Plant Fresh Weight (g) } \\
\hline & $\mathrm{g}^{-1}$ roots $(x 1000)$ & $\left(\mathrm{mL}^{-1}\right.$ soil $)$ & & Aerial Parts & Roots \\
\hline & & \multicolumn{4}{|c|}{ M. truncatula } \\
\hline 10 & $7.1 \pm 0.3$ & $14.2 \pm 0.4$ & $4.6 \pm 0.2$ & $33.2 \pm 1.2$ & $5.6 \pm 0.4$ \\
\hline 20 & $4.6 \pm 0.4$ & $6.8 \pm 0.2$ & $3.2 \pm 0.4$ & $45.4 \pm 0.9$ & $11.2 \pm 0.4$ \\
\hline 40 & $1.7 \pm 0.2$ & $2.4 \pm 0.2$ & $2.8 \pm 0.4$ & $60.6 \pm 0.9$ & $20.2 \pm 0.6$ \\
\hline Oxamyl (2 L ha $\left.{ }^{-1}\right)$ & $1.9 \pm 0.2$ & $5.2 \pm 0.4$ & $1.8 \pm 0.2$ & $42.0 \pm 0.7$ & $9.2 \pm 0.2$ \\
\hline Non treated & $11.7 \pm 0.7$ & $21.4 \pm 0.7$ & $4.8 \pm 0.2$ & $16.8 \pm 0.7$ & $2.6 \pm 0.2$ \\
\hline Non infested & - & & - & $52.2 \pm 1.8$ & $6.0 \pm 1.5$ \\
\hline LSD 0.05 & 0.9 & 1.1 & 0.9 & 3.4 & 1.8 \\
\hline
\end{tabular}

Soil treatments with the Medicago plant materials always resulted in a significant dose-related increase of tomato growth compared to the non-treated soil and, at the highest rate, even to Oxamyl and the non-infested control (Table 8).

\section{Discussion}

The saponin extract of the five Medicago species was demonstrated as strongly active on X. index and, except for M. hybrida, on M. incognita at concentrations above $250 \mu \mathrm{g} \mathrm{mL} \mathrm{L}^{-1}$, as confirming the almost complete mortality previously reported for a 16 or $24 \mathrm{~h}$ exposure of $M$ incognita J2 and X. index females to a $500 \mu \mathrm{g} \mathrm{mL}^{-1}$ solution of $M$. sativa saponins [26]. As in this cited study, G. rostochiensis J2 sensitivity to the five saponin mixtures was higher than that of M. incognita and X. index specimens, but lower than that previously showed to $M$. sativa saponins, which caused $40 \%-54 \%$ mortality even after a 4 and $8 \mathrm{~h}$ exposure, respectively, to a $125 \mu \mathrm{g} \mathrm{mL}^{-1}$ concentration [26]. Literature data on the activity of Medicago saponins on phytoparasitic nematodes are almost exclusively limited to the previous studies of our research group, which proved a biocidal activity of saponins, prosapogenins and sapogenins from M. arborea, M. arabica and M. sativa on X. index [25], as well as the activity of $M$. sativa saponin mixtures against the same nematode species tested in this study [26]. In addition, soil treatments with a crude extract of $M$. sativa saponins resulted in a significant reduction of $M$. incognita infestation on tomato [33]. More generally, only few data have been reported on the nematicidal effects of saponins from other plants. A significant effect on the motility of M. incognita J2 was reported for the saponins from Asparagus spp. [34], and saponin solutions were found to significantly reduce the number of $M$. javanica eggs and viable $J 2$ both in vitro and in soil [13]. Furthermore, the total saponin fraction of Portulaca oleracea L. and Lantana camara L. strongly reduced the in vitro motility of Meloidogyne spp. J2, as well as almost completely inhibited gall formation on eggplant roots under greenhouse conditions [35]. Adversely, the single saponin fraction of a $Q$. saponaria extract showed a poor nematicidal effect on a range of phytoparasitic nematodes, including $X$. index, the northern root-knot nematode M. hapla Chitwood, P. thornei, Tylenchorhynchus sp. and Helicotylenchus sp. [14].

Crude saponin content of the five Medicago species under investigation, as ranging from $0.60 \%$ of M. truncatula to $1.62 \%$ of $M$. heyniana, is in good agreement with published data, which reported a crude saponin content of about $0.5 \%-1.5 \%$ dry matter in medics [27,36-38]. The high molecular weight compounds detected at higher amounts in M. hybrida, M. lupulina and M. truncatula extracts, in particular saponins containing glycosides of medicagenic and zanhic acids, were previously identified in the aerial parts of Medicago species [8], including M. arborea [28], M. sativa [31], M. truncatula [37] and M. marina [32]. Given that zanhic acid is synthesized in the green parts of the plants [39], its glycosides have not previously been found in M. lupulina and M. hybrida $[8,38]$ when roots were investigated.

The biological activity of saponins is dependent on the number of side sugar chains attached to the sapogenins as well as to the nature of the sapogenin itself [8]. Thus, previous in vitro investigations allowed to relate the nematoxic effect of $M$. sativa saponins to the high amount of medicagenic acid. When pure aglycones have been used in in vitro bioassays, hederagenin was shown to be even 
more toxic than medicagenic acid and bayogenin against $X$. index, while soyasaponin $\mathrm{I}$, containing soyasapogenol B as a glycone, was the less-active saponin [25]. This finding seems to be confirmed also in the present study by the nematotoxic properties against $X$. index displayed by the three species $M$. heyniana, M. hybrida and M. murex, which produce saponins with high amounts of the two aglycones hederagenin and bayogenin. In addition, M. lupulina with a high amount of medicagenic acid was particularly active against $M$. incognita.

The mechanisms of the nematicidal activity of saponins are still not clearly elucidated, as different hypotheses were suggested by literature studies. Changes in cell permeability following the specific interaction of saponins with cell membranes were generally hypothesized as a cause of biological effects of these compounds [8,25]. More recently, Ibrahim and Srour [33] observed a decrease of cholesterol in eggs of root-knot nematodes, according to a concentration effect relationship, following soil treatments with a $M$. sativa saponin extract.

As suggested in our previous studies [25], a specific interaction of saponins with the nematode cuticle ultrastructure may be also involved in their mechanism of activity. Based on this hypothesis, differences in the cuticle chemical components of the nematode species could account for the different response of the three nematode species tested in this study to the five saponin mixtures [26].

Nevertheless, we have already noticed in a previous study [5] that the nematicidal activity of plant extracts and/or pure metabolites may vary according to the nematode species and the life stage of the same nematode species. Thus, for example, we have already observed that the potato cyst nematode G. rostiochiensis was more susceptible than $M$. incognita to the toxic effect of $A$. annua and its active compound, artemisinin [5]. Consistently, G. rostochiensis J2 was more sensitive to the five Medicago extracts than $M$. incognita and $X$. index also in the present study.

The effect of the five saponin extracts on the hatchability of M. incognita and G. rostochiensis eggs was the exact opposite, as the percentage hatch of $M$. incognita eggs was significantly reduced and did not affect or even stimulate the hatchability of $G$. rostochiensis eggs. Effects of saponins on nematode egg hatchability were scarcely documented, as literature studies mainly described their effects on $J 2$ motility and viability or on nematode infestation on host plants. The toxicity of the five saponin solutions to $M$. incognita eggs agrees with their effects on infective J2, whereas the stimulation of $G$. rostochiensis egg hatchability can be considered an unexpected result. An increased permeability of the cyst wall by the interaction with saponins may be hypothesized, though the exact biochemical mechanisms need to be specifically investigated.

The infestation of $M$. incognita on tomato plants was significantly reduced by soil amendments with the dry biomass of the five Medicago species, in full agreement with previous reports of a reduced infestation of M. incognita both on potted and field tomato or of the carrot cyst nematode Heterodera carotae Jones on field carrot following soil treatments with $M$. sativa biomass [40]. Moreover, previous studies also documented a significant suppression of phytoparasitic species M. javanica, Paratrichodorus sp. and Criconemella xenoplax joined to an increase of beneficial free-living nematodes, in a soil amended with M. sativa pelleted biomass [41], as well as an activity against a wide range of soilborne fungal strains of soil amendments with $M$. truncatula aerial parts [37].

Results from the in vitro experiments indicated that saponins from plant tissues of the five Medicago species are surely involved in the strong root-knot nematode suppression by soil incorporation of these plant materials. However, as already remarked in our previous studies [25,26], further contributory mechanisms should be also hypothesized, such as the nematoxicity of other bioactive metabolites from Medicago plants [42], an ammonia release by the decomposition of Medicago biomasses in soil [43,44] or an increase of phytonematode-suppressive microorganisms on the favorable substrate represented by Medicago tissues [45].

In particular it is known that Medicago species also synthesize polyphenolics such as glycosyl derivatives of apigenin, luteolin, chrysoeriol and tricin in M. truncatula [19,46] and in M. sativa [47,48]. Several biological functions related with plant structural protection, regulation of plant environmental communication and control of plant physiological events have been ascribed to plant polyphenolics. 
It has also been shown that they have a role as plant defense compounds against a range of microorganisms and that they are involved in plant-nematode interactions acting as defense compounds $[3,49,50]$. Thus, the presence of this type of metabolite in the biomass can reasonably be involved in the regulation of plant-nematode interactions when administered in soil to infested tomato plants.

The improved tomato growth in soil amended with the five Medicago species plant material also agrees with our previous reports of a biostimulating effect of soil incorporation with $M$. sativa biomass [42]. As previously noted for M. sativa, the growth effect of Medicago amendments may be related not only to the reduced nematode infestation but also to the physiological role of other specialized Medicago metabolites as well as to a general improvement of soil physical, chemical and microbiological properties such as an increased nitrogen content [51].

\section{Materials and Methods}

\subsection{Plant Material}

The five Medicago species, namely M. heyniana Greuter, M. hybrida (Pourr.) Trautv, M. lupulina L., M. murex Willd and M. truncatula Gaertn, were grown at the Institute of Soil Science and Plant Cultivation, Poland, and harvested at the beginning of flowering stage. Leaves were lyophilized, finely powdered and stored in dry conditions until use.

\subsection{Preparation of Saponin Extracts and Their Characterization}

Saponin extracts were prepared according to a standard procedure $[27,38]$. Briefly, the powdered leaves from each Medicago species (about $200 \mathrm{~g}$ ) were first defatted with $\mathrm{CHCl}_{3}$ in a Soxhlet apparatus and then saponins extracted with $80 \% \mathrm{MeOH}$ under reflux $(48 \mathrm{~h})$. After removing the solvent with a rotary evaporator, the residue of each extract was resuspended in $30 \% \mathrm{MeOH}$ and loaded onto a $\mathrm{C} 18$ column (Lichroprep RP-18, $50 \times 20$ mm, 40-63 $\mu \mathrm{m}$, Merck, Darmstad, Germany), equilibrated with MilliQ water. Elution was performed with $40 \% \mathrm{MeOH}(\mathrm{v} / \mathrm{v})$ to remove some polar compounds, and saponins were then eluted with $80 \% \mathrm{MeOH}(\mathrm{v} / \mathrm{v})$. Purified samples were then dried under reduced pressure. Saponin mixtures, obtained as brownish powder, were kept in airtight vials until used. Three extractions were performed on each sample and results expressed as mean \pm standard deviation (SD). The saponin mixtures were thus used for the successive analyses. Saponin extracts were checked by silica gel TLC plates, developed with ethyl acetate/acetic acid/water (7:2:2) and sprayed with the Liebermann-Burkhard reagent $(\mathrm{MeOH} /$ acetic anhydride/sulphuric acid, 10:1:1 v/v) to visualize the components.

\subsubsection{Hydrolysis of Saponins and Analysis of Sapogenins}

Crude saponin mix (10-15 mg) was treated with $30 \mathrm{~mL}$ of $2 \mathrm{~N} \mathrm{HCl}$ in $50 \%$ aqueous $\mathrm{MeOH}$ under reflux for $8 \mathrm{~h}$. After cooling, $\mathrm{MeOH}$ was eliminated under reduced pressure, $20 \mathrm{~mL}$ of water was added and then aglycones extracted with AcOEt $(2 \times 10 \mathrm{~mL})$. Each of the organic solutions containing the aglycones was dried by rotary evaporation and used in the successive analyses. Three independent hydrolysis reactions were performed for each sample.

Aglycone composition was determined by TLC, GC/FID and GC/MS methods by comparison to previously purified sapogenins from Medicago spp. [52]. Silica gel TLC was eluted with petroleum ether/ $\mathrm{CHCl}_{3} / \mathrm{AcOH}$ (7:2:1), and spots were visualized by spraying the developed TLC with the Liebermann-Burkhard reagent followed by heating at $120^{\circ} \mathrm{C}$. Sapogenins were also identified by GC/FID and GC/MS as their methyl-silyl derivatives. Samples were dissolved in $0.5 \mathrm{~mL}$ of $\mathrm{MeOH}$ and treated with $\mathrm{CH}_{2} \mathrm{~N}_{2}$. After solvent evaporation under a stream of nitrogen, silylation was performed by using $0.2 \mathrm{~mL}$ of a mixture of pyridine-hexamethyldisilazane-chlorotrimethylsilane $(2: 1: 1)$ at $70{ }^{\circ} \mathrm{C}$ for $10 \mathrm{~min}$. Reacted samples were properly diluted with isooctane and used for GC/FID and GC/MS analyses. Gas-chromatographic analyses were performed with a $30 \mathrm{~m} \times 0.32 \mathrm{~mm}, 0.25 \mu \mathrm{m}$ i.d., DB-5 
capillary column as described in Tava et al. [52]. Retention times and mass spectra data were compared to those of previously purified and identified sapogenins. The relative amount of each sapogenin was calculated as peak area percent relative to total peak area from GC/FID analysis of the whole saponin extract. For each Medicago species under investigation, results were expressed as the mean of three independent evaluations $\pm \mathrm{SD}$.

\subsubsection{LC/MS Analyses}

The investigated extracts were also subjected to UHPLC-ESI-MS analyses, using an ACQUITY UPLC chromatographic system (Waters, Milford, MA, USA), coupled with a PDA detector and a triple quadrupole mass detector (ACQUITY TQD, Waters). Saponins were separated on an ACQUITY HSS C18 column $(2.1 \times 100 \mathrm{~mm}, 1.8 \mu \mathrm{m}$; Waters $)$, maintained at $40{ }^{\circ} \mathrm{C}$; the injection volume was $2.5 \mu \mathrm{L}$. The elution method was: $0.0-0.5 \mathrm{~min}, 1 \%$ of solvent B (acetonitrile with $0.1 \%$ formic acid) in solvent $\mathrm{A}(0.1 \%$ formic acid in MilliQ water); 0.5-25.5 min, a linear gradient to 50\% B. The column was then washed with $99 \%$ B (2 min) and re-equilibrated with 1\% B (1.95 min) to return to the initial gradient. The flow rate was $0.400 \mathrm{~mL} \mathrm{~min}^{-1}$. The mass detector operated in negative ion mode. Capillary voltage was $3.0 \mathrm{kV}$; cone voltage was $40 \mathrm{~V}$; source temperature was $140{ }^{\circ} \mathrm{C}$; desolvation temperature was $350{ }^{\circ} \mathrm{C}$; desolvation gas flow was $800 \mathrm{~L} \mathrm{~h}^{-1}$; cone gas flow was $100 \mathrm{~L} \mathrm{~h}^{-1}$. Full-scan spectra were acquired in the range from 150 to $1600 \mathrm{~m} / \mathrm{z}$.

\subsection{Nematode Mortality Bioassays}

An Italian population of $M$. incognita was preliminarily multiplied on plants of tomato cv. Roma in a glasshouse maintained at $25 \pm 2{ }^{\circ} \mathrm{C}$ constant temperature. Formed egg masses were collected from the infested roots and incubated in distilled water in a growth chamber at $25^{\circ} \mathrm{C}$. An Italian population of G. rostochiensis Ro1 was reared on potato cv. Spunta in a glasshouse at $20^{\circ} \mathrm{C}$. Nematode cysts were extracted from the soil by Fenwick's flotation technique [53] and then incubated at $20^{\circ} \mathrm{C}$ in a $0.6 \mathrm{mM}$ sodium metavanadate hatching solution [54]. The emerged $J 2$ of both species were collected and stored in water at $5{ }^{\circ} \mathrm{C}$ until their use. The adult females of $X$. index were extracted from the soil of an infested vineyard located at Ginosa (Taranto province) by the Cobb's decanting and sieving method [55] and immediately used. All the three nematode species were preliminarily identified by a morphological characterization under an optical microscope. About $150 \mathrm{~J} 2$ of M. incognita or G. rostochiensis and 20 adult females of $X$. index were suspended in $0.5 \mathrm{~mL}$ distilled water in $1.5 \mathrm{~mL}$ Eppendorf vials. A $0.5 \mathrm{~mL}$ volume of 2000, 1000, 500 and $250 \mu \mathrm{g} \mathrm{mL}^{-1}$ aqueous solutions of the saponin extract of M. heyniana, M. truncatula, M. murex, M. hybrida and M. lupulina was added to each vial to obtain final concentrations of $1000,500,250$ and $125 \mu \mathrm{L} \mathrm{mL}^{-1}$. Nematodes were exposed to each concentration of the five saponin solutions for 4, 8, 16 or $24 \mathrm{~h}$, during which the Eppendorf tubes were maintained in agitation. There were four replicates for each concentration $x$ exposure time combination, and vials were arranged in a completely randomized experimental design. Distilled water and a $1 \mathrm{~mL} \mathrm{~L}^{-1}$ water solution of a liquid formulation ( $10 \%$ a.i.) of the nematicide Oxamyl were included as controls.

At the end of each exposure time, the nematodes from each replicate of each treatment were observed under a light microscope, assuming the complete immobility of M. incognita and G. rostochiensis $J 2$ and of needle-pricked X. index females [25] as evidence of the solution toxicity. The observed specimens were recovered on a $5 \mu \mathrm{m}$ sieve, repeatedly washed with water and then transferred to distilled water. Nematodes were considered dead if their immobility persisted after a $72 \mathrm{~h}$ permanence in water. Mortality rates were calculated according to Abbott's formula [55] $\mathrm{m}=100 \times(1-\mathrm{nt} / \mathrm{nc})$, in which $\mathrm{m}=$ percent mortality; $\mathrm{nt}=$ number of viable nematodes after the treatment; $\mathrm{nc}=$ number of viable nematodes in water. Two experimental runs each with a separate control were carried out on each nematode species. 


\subsection{Egg Hatchability Bioassays}

Groups of 50 M. incognita egg masses or G. rostochiensis cysts, averaging 400 eggs per mass and 700 eggs per cyst, respectively, were placed in $2 \mathrm{~cm}$ diameter sieves ( $215 \mu \mathrm{m}$ aperture) and submerged with $3 \mathrm{~mL}$ of the 500 and $1000 \mu \mathrm{L} \mathrm{ml}^{-1}$ solutions of the five saponin extracts within a $3.5 \mathrm{~cm}$ diameter Petri dish. The experiments on G. rostochiensis cysts were prepared in a $0.6 \mathrm{mM}$ water solution of sodium metavanadate, as reported as a hatching agent for this nematode species [54]. The egg masses and the cysts were exposed to each test solution for 1 or 2 weeks in a growth chamber at 25 and $20^{\circ} \mathrm{C}$, respectively. As in the first experiment, four replicates were provided for each treatment in comparison, arranging the experimental units in a complete randomized block design. Distilled water and pure $0.6 \mathrm{mM}$ sodium metavanadate solution were used as controls for egg masses and cysts, respectively, whereas the treatment with the same $1 \mathrm{~mL} \mathrm{~L}^{-1}$ water solution of Oxamyl solution used in the mortality assay was included as a chemical control. At the end of each exposure time, egg masses and cysts were removed from the test solutions and the hatching test continued in distilled water or in the $0.6 \mathrm{mM}$ sodium metavanadate solution, respectively. The emerged juveniles were removed and counted at weekly intervals. After each weekly removal of the emerged J2, egg masses and cysts were checked under a microscope, as to verify the occurrence of microbial contamination, and repeatedly washed with sterile water before renewing distilled water or $0.6 \mathrm{mM}$ sodium metavanadate solution. All these weekly operations were carried out under a laminar flow cabinet, as to avoid microbial contaminations potentially affecting the egg hatch. The $M$. incognita egg masses were removed from the sieves after a total of five weeks and then dissolved by a 3 min shaking in a $1 \%$ sodium hypochlorite aqueous solution [56]. The hatching test on G. rostochiensis was prolonged for eight weeks, after which cysts were crushed according to the Bijloo's modified method [57]. The unhatched eggs of both species were counted under a stereoscope. Egg hatchability was expressed as percentage ratio of total emerged J2 to the total egg content of egg masses or cysts. Both experiments were repeated twice with separate controls for each experiment.

\subsection{Experiment in Soil}

Roots of tomato cv Roma infested by the same population of $M$. incognita used for the experiments in vitro were minutely minced and thoroughly mixed. The number of eggs and $J 2$ per gram of roots was determined by processing six $10 \mathrm{~g}$ samples by the Hussey and Barker's method [56] and then counting the extracted eggs and $J 2$ under a microscope. A steam sterilised sandy soil $(64.4 \%$ sand, $18.7 \%$ silt, $16.9 \%$ clay, $0.8 \%$ organic matter and $7.5 \mathrm{pH}$ ) was added with appropriate amounts of the above infested roots, as to reach 8 eggs and $J 2 \mathrm{~mL}^{-1}$ initial nematode population density. Dry green biomass of the five Medicago species was thoroughly mixed to the infested soil at 10, 20 or $40 \mathrm{~g} \mathrm{~kg}^{-1}$ soil rates, and mixtures were poured into 1.2 L clay pots. Non-treated soil, either non-infested or infested by M. incognita, and soil treated with the liquid formulation of Oxamyl (10\% a.i.) used in the in vitro experiments, applied at an amount corresponding to a $2 \mathrm{~L} \mathrm{ha}^{-1}$ a.i. field rate three days before transplanting, were included as controls. Pots were arranged on greenhouse benches according to a randomized block design with five replicates of each treatment in comparison.

One tomato cv Tomito seedling ( 1 month old) was transplanted in each pot two weeks after soil amendments with the Medicago plant material. Tomato plants were maintained at a constant temperature of $25 \pm 2{ }^{\circ} \mathrm{C}$ throughout a two-month period, at the end of which fresh weight of aerial parts and roots was recorded on each replicate. Root gall infestation was estimated on each tomato plant according to the Taylor and Sasser's scale $(0=$ no galls, $1=1-2$ galls, $2=3-10$ galls, $3=11-30$ galls, $4=31-100$ galls and 5>100 galls) [58]. Final nematode population density was determined by extracting nematode eggs and $J 2$ from a $10 \mathrm{~g}$ sample of each tomato root [58] and from a $500 \mathrm{~mL}$ sample of soil from each pot [59]. 


\subsection{Statistical Analysis}

The arcsin transformed pooled data from the two experimental runs of the in vitro experiments and the $\operatorname{Ln}(x+1)$ transformed nematode data and raw plant growth data from the experiment in soil were subjected to one-way analysis of variance, comparing means by the Least Significant Difference Test at $p \leq 0.05$ [57]. The LC50 values of each saponin extract were also calculated with data from both in vitro assays by a probit-logistic analysis [55]. PlotIT 3.2 (Scientific Programming Enterprises, Haslett, MI, USA) software was used to perform all the stastistical analyses.

\section{Conclusions}

Results from this study demonstrate that saponin-rich extracts and plant biomasses from $M$. heyniana, M. hybrida, M. lupulina, M. murex and M. truncatula can be highly suppressive to root-knot nematodes and, therefore, could be included among the potential sources of new sustainable nematicidal products addressed to a management of phytoparasitic nematodes, also in a synergistic combination with other bio-derived products or other nonchemical techniques.

The presence on the market of products derived from saponin-rich extracts from Q. saponaria seems to demonstrate that an industrial exploitation of the five studied Medicago species may be technically and economically feasible, also due to the large biomass produced by these plants. Analogously, the already available nematicidal products based on dry biomasses of biofumigating Brassicaceae plants indicate potential granular or powdered formulations of the biomasses from the five Medicago species as a reasonable alternative to saponin extract-based products, also in consideration of the high suppressiveness demonstrated by soil amendments with the Medicago biomasses.

As generally remarked for plant-derived pesticides, an extended evaluation of the impact of potential Medicago plant-based products on other biotic soil components should be preliminarily undertaken, and agronomical techniques and plant-growing conditions should be preliminarily set up, as to avoid unstable effects on target nematodes related to a variable content of saponins and other bioactive compounds.

Author Contributions: Conceptualization, T.D. and P.A.; methodology, T.D., J.Ż.; software, T.D.; validation, M.P.A., J.Ż., A.T.; formal analysis, A.T., T.D., P.A.; investigation, J.Ż., M.P.A., E.B., T.D.; resources, X.X.; data curation, T.D., P.A., E.B., A.T.; writing—original draft preparation, T.D.; writing-review and editing, T.D., P.A., A.T.; visualization, T.D., P.A.; supervision, A.T., W.O.; funding acquisition, T.D., W.O. All authors have read and agreed to the published version of the manuscript.

Funding: This research received no external funding.

Acknowledgments: This study was carried out in the framework of a Bilateral Project between the Institute for Sustainable Plant Protection (CNR, Italy) and the Institute of Soil Science and Plant Cultivation Zoology (PAN, Poland).

Conflicts of Interest: The authors declare no conflict of interest.

\section{References}

1. Benelli, G. Plant-borne compounds and nanoparticles: Challenges for medicine, parasitology and entomology. Environ. Sci. Pollut. Res. 2018, 25, 10149-10150. [CrossRef] [PubMed]

2. Renčo, M.; Sasanelli, N.; Maistrello, L. Plants as natural sources of nematicides. In Nematodes: Comparative Genomics, Disease Management and Ecological Importance; Davis, L.M., Ed.; NOVA Science Publisher: New York, NY, USA, 2014; pp. 115-141.

3. Ntalli, N.G.; Caboni, P. Botanical nematicides: A review. J. Agric. Food Chem. 2012, 60, 9929-9940. [CrossRef] [PubMed]

4. Avato, P.; D'Addabbo, T.; Leonetti, P.; Argentieri, M.P. Nematicidal potential of Brassicaceae. Phytochem. Rev. 2013, 12, 791-802. [CrossRef]

5. D'Addabbo, T.; Carbonara, T.; Argentieri, M.P.; Radicci, V.; Leonetti, P.; Villanova, L.; Avato, P. Nematicidal potential of Artemisia annua and its main metabolites. Eur. J. Plant Pathol. 2013, 137, 295-304. [CrossRef] 
6. Laquale, S.; Avato, P.; Argentieri, M.P.; Bellardi, M.G.; D'Addabbo, T. Nematotoxic activity of essential oils from Monarda species. J. Pest Sci. 2018, 91, 1115-1125. [CrossRef]

7. Avato, P.; Laquale, S.; Argentieri, M.P.; Lamiri, A.; Radicci, V.; D'Addabbo, T. Nematicidal activity of essential oils from aromatic plants of Morocco. J. Pest Sci. 2017, 90, 711-722. [CrossRef]

8. Tava, A.; Avato, P. Chemical and biological activity of triterpene saponins from Medicago species. Nat. Prod. Commun. 2006, 1, 1159-1180. [CrossRef]

9. Güçlü-Ustündağ, O.; Mazza, G. Saponins: Properties, applications and processing. Crit. Rev. Food Sci. Nutr. 2007, 47, 231-258. [CrossRef]

10. Maestrini, M.; Tava, A.; Mancini, S.; Salari, F.; Perrucci, S. In vitro anthelmintic activity of saponins derived from Medicago spp. plants against donkey gastrointestinal nematodes. Vet. Sci. 2019, 6, 35. [CrossRef]

11. Maestrini, M.; Tava, A.; Mancini, S.; Tedesco, D.; Perrucci, S. In vitro anthelmintic activity of saponins from Medicago spp. against sheep gastrointestinal nematodes. Molecules 2020, 25, 242. [CrossRef]

12. Chitwood, D.J. Phytochemical based strategies for nematode control. Ann. Rev. Phytopathol. 2002, 40, 221-249. [CrossRef] [PubMed]

13. Omar, S.A.; Abdel-Massih, M.I.; Mohamed, B.E. Use of saponin to control the root-knot nematode Meloidogyne javanica in tomato plants. Bull. Fac. Agric. Cairo 1994, 45, 933-940.

14. Magunacelaya, J.C.; San Martín, R. Control of plant-parasitic nematodes with extracts of Quillaja saponaria. Nematology 2005, 7, 577-585. [CrossRef]

15. D'Addabbo, T.; Curto, G.; Greco, P.; Di Silvestro, D.; Coiro, M.I.; Lamberti, F.; Ferrari, V.; Santi, R.; Carella, A. Prove preliminari di lotta contro nematodi galligeni mediante estratti di Quillaja saponaria Molina. Nematol. Medit. 2005, 33, 29-34.

16. Barnes, J.; Anderson, L.A.; Phillipson, J.D.; Newall, C.A. Herbal Medicines, 3rd ed.; Pharmaceutical Press: London, UK, 2007; pp. 176-213.

17. Stochmal, A.; Oleszek, W. Seasonal and structural changes of flavones in alfalfa (Medicago sativa) aerial parts. J. Food Agric. Environ. 2007, 5, 84-88.

18. Golawska, S.; Lukasik, I.; Kapusta, I.; Janda, B. Do the content of luteolin, tricin and chrysoeriol glycosides in alfalfa (Medicago sativa L.) affect the behaviour of pea aphid (Acyrthosiphon pisum)? Pol. J. Environ. Stud. 2012, 21, 1613-1619.

19. Kowalska, I.; Stochmal, A.; Kapusta, I.; Janda, B.; Pizza, C.; Piacente, S.; Oleszek, W. Flavonoids from barrel medic (Medicago truncatula) aerial parts. J. Agric. Food Chem. 2007, 55, 2645-2652. [CrossRef]

20. Tava, A.; Mella, M.; Avato, P.; Argentieri, M.P.; Bialy, Z.; Jurzysta, M. Triterpenoid glycosides from leaves of Medicago arborea L. J. Agric. Food Chem. 2005, 53, 9954-9965. [CrossRef]

21. Bialy, Z.; Jurzysta, M.; Mella, M.; Tava, A. Triterpene saponins from aerial parts of Medicago arabica L. J. Agric. Food. Chem. 2004, 52, 1095-1099. [CrossRef]

22. Avato, P.; Migoni, D.; Argentieri, M.P.; Tava, A.; Fanizzi, F.P. Activity of saponins from Medicago species against HeLa and MCF-7 cell lines and their capacity to potentiate cisplatin effect. Anticancer Ag. Med. Chem. 2017, 17, 1508-1518. [CrossRef]

23. Saniewska, A.; Jarecka, A.; Bialy, Z.; Jurzysta, M. Antifungal activity of saponins originated from Medicago hybrida against some ornamental plant pathogens. Acta Agrobot. 2006, 59, 51-58. [CrossRef]

24. Abbruscato, A.; Tosi, S.; Crispino, L.; Biazzi, E.; Menin, B.; Picco, A.M.; Pecetti, L.; Avato, P.; Tava, A. Triterpenoid glycosides from Medicago sativa as anti fungal agents against Pyricularia oryzae. J. Agric. Food Chem. 2014, 62, 11030-11036. [CrossRef] [PubMed]

25. Argentieri, M.P.; D’Addabbo, T.; Tava, A.; Agostinelli, A.; Jurzysta, M.; Avato, P. Evaluation of nematicidal properties of saponins from Medicago spp. Eur. J. Plant Pathol. 2008, 120, 189-197. [CrossRef]

26. D'Addabbo, T.; Avato, P.; Tava, A. Nematicidal potential of materials from Medicago spp. Eur. J. Plant Pathol. 2009, 125, 39-49. [CrossRef]

27. Tava, A.; Pecetti, L. Chemical investigation of saponins from twelve annual Medicago species and their bioassay with the brine shrimp Artemia salina. Nat. Prod. Comm. 2012, 7, 837-840. [CrossRef]

28. Tava, A.; Mella, M.; Avato, P.; Biazzi, E.; Pecetti, L.; Bialy, Z.; Jurzysta, M. New Triterpenic Saponins from the aerial parts of Medicago arabica (L.) Huds. J. Agric. Food Chem. 2009, 57, 2826-2835. [CrossRef]

29. Tava, A.; Scotti, C.; Avato, P. Biosynthesis of saponins in the genus Medicago. Phytochem. Rev. 2011, 10, 459-469. [CrossRef] 
30. Tava, A.; Pecetti, L.; Romani, M.; Mella, M.; Avato, P. Triterpenoid glycosides from the leaves of two cultivars of Medicago polymorpha L. J. Agric. Food Chem. 2011, 59, 6142-6149. [CrossRef]

31. Bialy, Z.; Jurzysta, M.; Oleszek, W.; Piacente, S.; Pizza, C. Saponins in alfalfa (Medicago sativa) root and their structural elucidation. J. Agric. Food Chem. 1999, 47, 3185-3192. [CrossRef]

32. Tava, A.; Biazzi, E.; Ronga, D.; Mella, M.; Doria, F.; Accogli, R.; Argentieri, M.P.; Avato, P. Triterpenic saponins from Medicago marina L. Phytochemistry 2020, 174, 112333. [CrossRef]

33. Ibrahim, M.A.; Srour, H.A. Saponins suppress nematode cholesterol biosynthesis and inhibit root knot nematode development in tomato seedlings. Nat. Prod. Chem. Res. 2013, 2, 1-4. [CrossRef]

34. Meher, H.C.; Walia, S.; Sethi, C.L. Effect of steroidal saponins on the mobility of juveniles of Meloidogyne incognita. Ind. J. Nematol. 1988, 18, 244-247.

35. Ibrahim, H.S.; Hamouda, S.E.S.; El-Kady, A.M.A.; Abd-Alla, H.I. Study the nematicidal efficiency of Corchorus olitorius, Cinnamomum camphora, Portulace oleracea and Lantana camara extracted saponins and their formulations on root-knot nematodes Meloidogyne spp. Nat. Sci. 2014, 12, 40-45.

36. Huhman, D.V.; Berhow, M.A.; Sumner, L.W. Quantification of saponins in aerial and subterranean tissues of Medicago truncatula. J. Agric. Food Chem. 2005, 53, 1914-1920. [CrossRef] [PubMed]

37. Kapusta, I.; Stochmal, A.; Perrone, A.; Piacente, S.; Pizza, C.; Oleszek, W. Triterpene saponins from barrel medic (Medicago truncatula) aerial parts. J. Agric. Food Chem. 2005, 53, 2164-2170. [CrossRef] [PubMed]

38. Bialy, Z.; Jurzysta, M.; Mella, M.; Tava, A. Triterpene saponins from the roots of Medicago hybrida. J. Agric. Food Chem. 2006, 54, 2520-2526. [CrossRef]

39. Carelli, M.; Biazzi, E.; Tava, A.; Losini, I.; Abbruscato, P.; Depedro, C.; Scotti, C. Sapogenin content variation in Medicago inter-specific hybrid derivatives highlights some aspects of saponin synthesis and control. New Phytol. 2015, 206, 303-314. [CrossRef]

40. D'Addabbo, T.; Carbonara, T.; Leonetti, P.; Radicci, V.; Tava, A.; Avato, P. Control of plant parasitic nematodes with active saponins and biomass from Medicago sativa. Phytochem. Rev. 2011, 10, 503-519. [CrossRef]

41. Walker, G.E. Effects of organic amendments, fertilisers and fenamiphos on parasitic and free-living nematodes, tomato growth and yield. Nematol. Medit. 2007, 35, 131-136.

42. Natelson, S. Canavanine in alfalfa (Medicago sativa). Experientia 1985, 41, 257-259. [CrossRef]

43. Bailey, K.L.; Lazarovitis, G. Suppressing soil-borne diseases with residue managment and organic amendments. Soil Tillage Res. 2003, 72, 169-180. [CrossRef]

44. Jansen, H.H.; McGinn, S. Volatile loss of nitrogen during decomposition of legume green manure. Soil Biol. Biochem. 1991, 23, 291-297. [CrossRef]

45. Jaffee, B.A. Interactions among a soil organic amendment, nematodes, and the nematode-trapping fungus Dactylellina candidum. Phytopathology 2006, 96, 1388-1396. [CrossRef] [PubMed]

46. Marczak, Ł.; Stobiecki, M.; Jasinski, M.; Oleszek, W.; Kachlicki, P. Fragmentation Pathways of acylated flavonoid diglucuronides from leaves of Medicago truncatula. Phytochem. Anal. 2010, 21, 224-233. [CrossRef]

47. Stochmal, A.; Piacente, S.; Pizza, C.; De Riccardis, F.; Leitz, R.; Oleszek, W. Alfalfa (Medicago sativa L.) flavonoids. 1. Apigenin and luteolin glycosides from aerial parts. J. Agric. Food Chem. 2001, 49, 753-758. [CrossRef]

48. Stochmal, A.; Simonet, A.M.; Macias, F.A.; Oleszek, W. Alfalfa (Medicago sativa L.) flavonoids. 2. Tricin and chrysoeriol glycosides from aerial parts. J. Agric. Food Chem. 2001, 49, 5310-5314. [CrossRef]

49. Falcone Ferreyra, M.L.; Rius, S.P.; Casati, P. Flavonoids: Biosynthesis, biological functions, and biotechnological appliocations. Front. Plant Sci. 2012, 3. [CrossRef]

50. Chin, S.; Behm, C.A.; Mathesius, U. Functions of flavonoids in plant-nematode interactions. Plants 2018, 7, 85. [CrossRef]

51. Bulluck, L.R.; Brosius, M.; Evanylo, G.K.; Ristaino, J.B. Organic and synthetic fertility amendments influence soil microbial, physical and chemical properties on organic and conventional farms. Appl. Soil Ecol. 2002, 19, 147-160. [CrossRef]

52. Tava, A.; Biazzi, E.; Mella, M.; Quadrelli, P.; Avato, P. Artefact formation during acid hydrolysis of saponins from Medicago spp. Phytochemistry 2017, 238, 116-127. [CrossRef]

53. Hooper, D.J.; Hallmann, J.; Subottin, A.S. Methods for Extraction, Processing and Detection of Plant and Soil Nematodes. In Plant Parasitic Nematodes in Subtropical and Tropical Agriculture, 2nd ed.; Luc, M., Sikora, R.A., Bridge, J., Eds.; CABI Publishing: Wallingford, UK, 2005; pp. 53-86. 
54. Clarke, A.J.; Shepherd, A.M. Hatching agents for the potato cyst-nematode, Heterodera rostochiensis Woll. Ann. Appl. Biol. 1968, 61, 139-149. [CrossRef]

55. Finney, D.J. Statistical Method in Biological Assay, 3rd ed.; Charles Griffin \& Company Ltd.: High Wycombe, UK, 1978; p. 508.

56. Hussey, R.S.; Barker, K.R. A comparison of methods of collecting inocula of Meloidogyne spp. including a new technique. Plant Dis. Rep. 1973, 57, 1025-1028.

57. Den Ouden, H.; Seinhorst, J.W. An improvement of Bijloo's method for determining the egg content of Heterodera cysts. Nematologica 1966, 12, 170-171. [CrossRef]

58. Taylor, A.L.; Sasser, J.N. Biology, Identification and Control of Root-Knot Nematodes (Meloidogyne spp.); North Carolina State University: Raleigh, NC, USA, 1978; p. 111.

59. Coolen, W.A. Methods for the extraction of Meloidogyne spp. and other nematodes from roots and soil. In Root-Knot Nematodes (Meloidogyne species): Systematics, Biology and Control; Lamberti, F., Taylor, C.E., Eds.; Academic Press: London, UK, 1979; pp. 317-329.

(C) 2020 by the authors. Licensee MDPI, Basel, Switzerland. This article is an open access article distributed under the terms and conditions of the Creative Commons Attribution (CC BY) license (http://creativecommons.org/licenses/by/4.0/). 